\title{
On the Mechanisms Underlying the Secretion and Export of Translation- ally Controlled Tumor Protein/Histamine Releasing Factor (TCTP/HRF)
}

\author{
Jeehye Maeng, Miyoung Kim and Kyunglim Lee* \\ College of Pharmacy, Center for Cell Signaling \& Drug Discovery Research, Ewha Womans University, Seoul 120-750, \\ Korea
}

\begin{abstract}
Numerous studies have demonstrated that TCTP/HRF is a unique cytokine, modulating the release of inflammatory mediators from various cell types including cells involved in allergic phenomena. Despite the absence of a leader sequence in its $\mathrm{NH}_{2}$-terminus, TCTP/HRF is regarded as a secreted protein found outside of cells as well as in fluids from allergic patients and parasitic organisms. Recent studies clarified several potential mechanisms leading to its secretion. For example, these studies showed that TCTP/HRF is exported from cells via a non-classical, endoplasmic reticulum (ER)/Golgi-independent mechanism associated with exosomal transport. TSAP6, a p53-inducible transmembrane protein, has been shown to enhance exosome production, and facilitate the secretion of TCTP/HRF into extracellular milieu. Additionally, H,K-ATPase also appears to play a role in the transport of TCTP/HRF, since inhibitors of H,K-ATPase also inhibit TCTP/HRF exit. The exact mechanisms involved in TCTP/HRF secretion has not yet emerged. Here we attempted to collate the available information in the current understanding of the mechanisms underlying the release of TCTP/HRF and of the factors that seem to influence these mechanisms.
\end{abstract}

Keywords: Allergy, histamine releasing factor (HRF), H,K-ATPase, proton pump inhibitor, secretion, translationally controlled tumor protein (TCTP), TSAP6.

\section{INTRODUCTION}

Translationally controlled tumor protein (TCTP), also known as histamine releasing factor (HRF) or fortilin, (hereafter called TCTP/HRF in this review), is a highly conserved protein, ubiquitously expressed in almost all eukaryotic organisms [1]. TCTP/HRF has a structural homology with the small chaperone family of Mss4/Dss4 proteins which are reported to bind to the nucleotide-free form of Rab proteins [2]. Their tubulin-binding domain and the $\mathrm{Ca}^{2+}$-binding region are mapped [3, 4]. Intracellular expression of TCTP/HRF is regulated at both the transcriptional and translational levels by a wide range of extracellular signals and stimuli [1]. TCTP/HRF plays roles in cell cycle progression [3], malignant transformation [5], and anti-apoptotic activity [6] in addition to its extracellular, cytokine-like function as a histamine-releasing factor $[7,8]$ with potential implications for roles in immune responses and allergy. In addition, our group showed that TCTP/HRF plays several previously unrecognized functional roles. One is its function as a cytoplasmic repressor of $\mathrm{Na}, \mathrm{K}-\mathrm{ATPase}$ [9] because of which, overexpression of TCTP/HRF in vivo leads to hypertension, and increases intracellular calcium mobilization [10]. Another new finding on TCTP/HRF we recently reported is that its amino terminal region functions as a protein transduction domain (PTD) [11]. As is well known, PTD peptides are used to enhance cellular uptake of drugs, proteins, polynucleotides or liposomes [12, 13]. Extracellularly secreted

*Address correspondence to this author at the College of Pharmacy, Center for Cell Signaling \& Drug Discovery Research, Ewha Womans University, Seoul 120-750, Korea; Tel: +82 23277 3024; Fax: +82 23277 2851;

E-mail: klyoon@ewha.ac.kr
TCTP/HRF is modified and activated by dimerization, to acquire cytokine-like function [7]. A peptide binding to dimerized TCTP/HRF inhibits the release of inflammatory mediators in vivo [14].

Despite the plethora of reports on TCTP/HRF, including its ubiquitous distribution, its conservation in various species, and its influence on a variety of biological phenomena, a unified concept of the primary functional role of TCTP/HRF has not yet emerged. This review will briefly highlight recent advances in our understanding of the secretion of TCTP/HRF, and of the possible underlying mechanisms. It will not cover, except when necessary for this narrative, topics such as historical aspects of the discovery and early researches on TCTP/HRF, including its animal and plant variants, structures, functions, and interactions, and transgenic studies which will be covered in other chapters.

Secretions of TCTP/HRF have also been shown to be present in activated mononuclear cells, platelets, in nasal lavages, skin blister fluids, and bronchoalveolar lavage (BAL) fluids obtained during late phase allergic reaction [15-18]. TCTP/HRF is now regarded as a protein secreted from erythroid and leukemic cells as well as bronchial epithelial cells (Table 1), which suggests that TCTP/HRF may also be secreted by other cell types yet to be identified $[8,16,19-23]$.

TCTP/HRF was originally viewed as a secretagogue of histamine and interleukin (IL)-4 from basophils in donors having particular type of IgE [24]. Subsequent studies revealed that regardless of the type of IgE, TCTP/HRF can enhance IgE-dependent histamine release as well as IL-4 and IL-13 production by basophils [25]. In fact, it has actually 
Table 1. Secretion of TCTP/HRF from Different Cell Types

\begin{tabular}{|c|c|c|}
\hline Cell Types & Description & References \\
\hline \hline Epithelial cells & & \\
\hline 293T cells & Human embryonic kidney cells & {$[19]$} \\
\hline HEK293 cells & Human embryonic kidney cells & {$[20]$} \\
\hline HepG2 cells & $\begin{array}{c}\text { Human hepatocellular carcinoma } \\
\text { cells }\end{array}$ & {$[19]$} \\
\hline BEAS-2B cells & Human bronchial epithelial cells & {$[23]$} \\
\hline Hematopoietic cells & Human macrophage cells & {$[8,20]$} \\
\hline U937 cells & Murine peritoneal macrophages & {$[22]$} \\
\hline Murine macrophages & Peripheral blood mononuclear & {$[8]$} \\
\hline cells & {$[19]$} \\
\hline K562 cells & Human erythroleukemia cells & {$[19]$} \\
\hline D1 cells & Murine dendritic cells & {$[21]$} \\
\hline Jurkat cells & Human T cell leukemia cells & {$[21]$} \\
\hline BJAB cells & Human B cell lymphoma cells & \\
\hline
\end{tabular}

been suggested that TCTP/HRF interacts with its own receptor and exerts cytokine-like activity, and not through $\operatorname{IgE}$ or FceRI [26]. TCTP/HRF also activates chemotaxis, triggers IL-8 release from eosinophils and induces intracellular calcium mobilization [27]. In addition, TCTP/HRF is a B cell growth factor inducing cytokine production from these cells [28]. Yoneda et al., reported that TCTP/HRF also stimulates non-immune, bronchial epithelial cells to produce IL-8 and granulocyte/macrophage colony-stimulating factor (GMCSF) [23]. These functions including the promotion of the production of interleukins from basophils and eosinophils $[25,27]$, make TCTP/HRF an important player facilitating general cytokine-like activities involved in immune response. Although several aspects of secretion of TCTP/HRF have been well documented, how TCTP/HRF is eventually exported out of cells to promote histamine release is not yet fully understood.

\section{SECRETION OF TCTP/HRF AND THE UNDERLY- ING MECHANISMS}

It is well established that secretion of TCTP/HRF into biological fluids influences a multitude of biological and cellular phenomena. However, there is no clear understanding of the factors contributing to the release and export of TCTP/HRF. In general, secreted cellular proteins are typically exported by one of two major routes. A majority of secreted proteins which possess signal sequences composed of 13-30 hydrophobic amino acids at their $\mathrm{NH}_{2}$-termini, exit via an ER/Golgi-dependent pathway. This classical secretion is mediated by a leader sequence that directs the protein through the ER/Golgi-dependent route, followed by vesicular transport to the biological membrane, and extracellular release of the protein in a soluble form. Some intracellular proteins are resistant to inhibitors of ER/Golgi trafficking such as brefeldin A or monensin [29, 30], and are exported without signal peptide, independent of the ER/Golgi apparatus, via secretory pathway, that is called an alternative pathway or unconventional secretion [31]. IL-1 $\beta$ and galectin-1 were the first leaderless secretory proteins that were shown to follow such ER/Golgi-independent route [32, 33]. Such unconventional export can be mediated by several potential mechanisms, which involve exosomes, lysosomal secretion, plasma membrane resident transporters, and exovesicles or membrane blebbing [34]. Exosomes which are one of the agents that mediate protein export are small vesicular organelles that are secreted as a consequence of fusion of multivesicular late endosome with the plasma membrane [35], leading to release of exosome-loaded cargo into the extracellular space [36]. Exosomes are responsible for the export of major histocompatibility (MHC) class I and II, and integrin family through the non-classical pathway [31, 37-39], which is believed to be associated with several different physiological processes [40].

Since TCTP/HRF proteins lack any obvious secretion signaling residues at their $\mathrm{NH}_{2}$-termini and no precursor protein has been described [41], these proteins may be secreted by unconventional routes, via ER/Golgi-independent fashion such as that described for fibroblast growth factor (FGF)-1, FGF-2 [42, 43], thioredoxin [44], lipocortin, alternatively called annexin [45], galectin [46], HIV-Tat protein [47], plasminogen activator inhibitor 2 [48] and IL-1 [44, 49]. It is therefore logical to suggest that TCTP/HRF, which also has no classical leader sequence, exits from cells via an alternative or unconventional pathway, e.g. through lysosomal and exosomal secretion, plasma membrane resident transporters, and perhaps other pathways [31, 34, 50, 51]. A definitive mechanism for the secretion of leaderless TCTP/HRF proteins was first unveiled by Amzallag et al [19]. They suggested that TCTP/HRF is secreted by an ER/Golgi-independent, exosomal route and described the underlying mechanisms. Recently, our laboratory proposed the involvement of H,K-ATPase [20], in TCTP/HRF secretion and this finding was corroborated by the observation that H,K-ATPase inhibitor ameliorated the allergic symptoms. Several stimulators such as $\mathrm{H}_{2} \mathrm{O}_{2}$ [23], M-CSF [22], and dioxin [21] have also been found to promote the secretion of TCTP/HRF homologues, although other cytokines or factors, as yet undefined, may also play pivotal roles in TCTP/HRF secretion. A summary of publications on TCTP/HRF secretion is presented in Fig (1). Clearly, a unifying picture of the mechanisms involved in TCTP/HRF secretion is yet to emerge.

The vast body of information that has accumulated on the pathways of TCTP/HRF secretion and the underlying pathophysiological mechanisms, suggests that secretion of TCTP/HRF occurs via ER/Golgi-independent, non-classical pathway operating in the exosome. Secretion of TCTP/HRF regulated either a) by a tumor suppressor activated pathway6 (TSAP6)-mediated [19] route, or b) by via a H,K-ATPasemediated [20] route.

\section{a) ER/Golgi-independent, Exosomal Export of TCTP/HRF via TSAP6-mediated Pathway}

How TCTP/HRF is eventually exported out of cells to promote inflammatory responses became clear only after the finding that TSAP6, a multi-transmembrane protein, modifies the trafficking and regulates exosomal secretion of TCTP/HRF [19]. Of note, it was shown that overexpression 


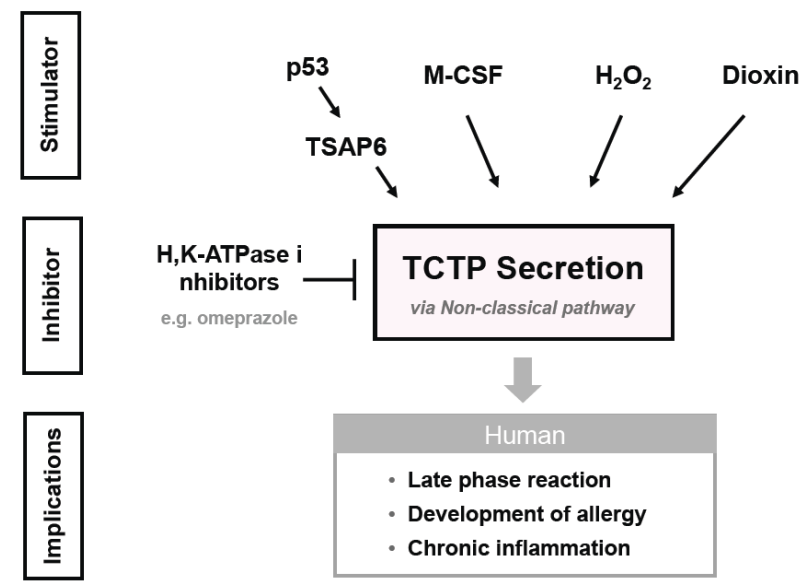

Fig. (1). A Schematic Summary of Secretion of TCTP/HRF TCTP/HRF is secreted via ER/Golgi-independent, non-classical pathway involving exosomal export. Secretion of TCTP/HRF is triggered by p53-inducible TSAP6, M-CSF, and agents such as $\mathrm{H}_{2} \mathrm{O}_{2}$ and dioxin. $\mathrm{H}, \mathrm{K}-\mathrm{ATP}$ ase inhibitors including omeprazole and pantoprazole inhibit TCTP/HRF release in vitro and in vivo. Secreted TCTP/HRF homologues modulate a series of immune responses via extracellular, cytokine-like activities in humans.

of TSAP6, facilitates the exit of TCTP/HRF via exosomal route and that TSAP6 plays a role in either the selective transport of specific proteins to the exosome, or in the regulation of exosome production $[19,52]$. TSAP6, a p53inducible ferrireductase [53], and human homologue of the rat pHyde [54], was originally discovered as a transcript upregulated by p53 [55], and is reportedly involved in apoptosis, and cell cycle regulation [56]. TSAP6-related proteins such as STAMP1 and TIARP1 have been localized to vesicular structures and have been implicated in vesicular trafficking and secretory processes $[57,58]$. Secretion of either endogenously or exogenously expressed TCTP/HRF was not hampered either by brefeldin A or monensin treatments, suggesting that they proceed via an ER/Golgi-independent or nonclassical pathway [19]. A functional relationship between TSAP6 and TCTP/HRF was confirmed by the observation that overexpression of TSAP6 considerably enhanced the secretion of both endogenous and exogenous TCTP/HRF in epithelial cells as well as in hematopoietic cell lines. Furthermore, both TSAP6 and TCTP/HRF were co-localized in the vesicular-like structures, and TCTP/HRF was found in exosomal preparations derived from supernatants of dendritic cells, suggesting that TCTP/HRF may be secreted via exosomal export [19]. It is also indicated that TCTP/HRF is associated with TSAP6 in the cytoplasm as well as in exosomes and the intracellular content of TCTP/HRF increased in Tsap6-null cells [19, 52]. Interestingly, nonclassically exported, leaderless galectin-3 was also found in exosome preparations from dendritic cells [59] together with TCTP/HRF in the same sucrose gradient fraction [19], suggesting that both these proteins may exert their extracellular activities on neighboring cells via exosomes involved in intercellular communications [39]. In accord with the finding that TSAP6 increased TCTP/HRF in the supernatants, TSAP6 also raised TCTP/HRF levels in exosomes derived from 293T cells. These observations confirm the role of TSAP6 in the export of TCTP/HRF [19].
TSAP6 also seems to play a role in the selective transport of proteins of exosomes, as shown by the finding that TSAP6 specifically enhanced the presence of MHC class I, but not that of clathrin in exosomes [19]. Since dendritic cell-derived exosomes include both MHC class I and II and have been shown to induce potent immune responses [38, 59], exosome-loaded TSAP6 is possibly involved in exosome-associated activities. Notably, there is robust evidence that exosomes are readily secreted by certain immune cells including cytotoxic $\mathrm{T}$ cells, for specifically targeting mediators of cytolysis, and B cells and dendritic cells, for antigen presentation and $\mathrm{T}$ cell proliferation, respectively $[35,60]$. Therefore, exosomes are likely to affect a series of immune responses. The majority of secreted TCTP/HRF appeared to originate from pre-existing intracellular pools, rather than from newly synthesized proteins, and TCTP/HRF has a relatively long half-life [19]. It was suggested that a non-secretable pool of TCTP/HRF contributing to various intracellular functions exists, and this explains why only small fractions of the total intracellular TCTP/HRF is released. In this regard, it was speculated that pre-existing pools of TCTP/HRF can act as a readily accessible store enabling the rapid recruitment of pre-packaged mediators to the site of allergic inflammation upon stimulation [19]. Taken together, these observations suggest that TSAP6 plays novel roles facilitating export of TCTP/HRF, and that it takes part in the regulation of vesicular trafficking and exosomal secretion. Recently, a model was proposed for the regulation of the secretion of exosomes by TSAP6, using TSAP6/Steap3-null mice [52].

These concepts on the regulatory mechanism for exosomal export were further extended by invoking a novel role for p53 in regulating exosome secretion in conjunction with activation of TSAP6 [61]. p53 responds to various stress signals such as genotoxins, hypoxia, and activated oncogenes [62] by regulating the transcription of target genes. Since TSAP6 is known as a p53-regulated gene product and is transcribed in response to stress conditions [56], p53 is possibly involved in exosome production through upregulation of TSAP6 transcription in response to DNA damage. Employing a proteomic approach to investigate this hypothesis, proteins secreted from cells, and existing in exosomes after p53-mediated stress response of cells, were identified. Activation of p53 facilitates export of non-transcriptional targets of p53 via exosomes, and exosome production by cells is regulated by the p53 response [61]. This was supported by the finding that exosome secretion which normally occurs at a low rate in many cells, is significantly enhanced when p53 is activated [61]. Since TSAP6 enhances exosome production in cells reacting with a p53 response to stress, it has been suggested that p53 mediates enhanced exosome production and secretion by transcribing the TSAP6 gene and causing the production of TSAP6 [61]. Thus the regulation of the production of exosomes, and the exosomal secretion of TCTP/HRF seem to be controlled by TSAP6 and p53.

These findings showing that TCTP/HRF is secreted through exosomes which are regulated by TSAP6 and p53 $[19,61]$, and that TSAP6 regulates apoptosis and cell cycle progression [56], providing the connection between TSAP6 and tumor reversion as suggested by Telerman and Amson [63]. These authors developed a series of biological models 
to show how a tumor cell sheds its malignant status and becomes non-malignant and that TCTP/HRF and TSAP6 are the key genes in this regard [63]. Also, it was previously shown that TSAP6 is upregulated in the wild-type p53 cells or reverted cells from malignant state, while TCTP/HRF is repressed in those cells $[5,55,56]$. These authors speculated that TSAP6 regulates exosome production to expel proteins that are no longer necessary for their survival and TCTP/HRF exemplifies proteins expelled from cells through interaction with TSAP6. In this regard, exosome may play the role of 'detoxifier' of the cells in a p53-regulated process [52]. Thus, the function of TSAP6, in the context of molecular pathways of tumor reversion, has been conceptualized as a 'detoxifier' [63]. Since TSAP6 exports proteins that are dispensable for normal cell development and survival, tumor cells get rid of survival factors such as TCTP/HRF as they undergo reversion, by activating the TSAP6 [63]. In addition to TCTP/HRF, TSAP6 which is also upregulated during the reversion process initiated by $\mathrm{p} 53$ gene, is considered to be necessary for tumor reversion, because of its ability to promote the expulsion of TCTP/HRF from cells, thereby lowering its concentration of TCTP/HRF-overexpressing cancerous cells. These authors indicate that some antidepressive and neuroleptic drugs exert anti-tumor effects by directly binding to TCTP/HRF and interrupting the interaction with partner proteins, thereby promoting its export and lowering its intracellular concentration [63]. Overall, this model offers a unique view of the role of TSAP6-regulated secretion of proteins in inhibiting tumors through exosomal exit and reorganization of tumor cell growth by lessening the cytoplasmic burden of TCTP/HRF.

\section{b) H,K-ATPase-mediated pathway of TCTP/HRF secretion}

Our group provided the evidence for the involvement of H,K-ATPase in the secretory pathway of TCTP/HRF [20]. To gain insights into the secretion of TCTP/HRF, we investigated whether agents that inhibit release of TCTP/HRF can serve as anti-allergic asthmatic drugs [20]. Previous studies showing that secretion of the FGF-2 is mediated by the catalytic subunit of $\mathrm{Na}, \mathrm{K}-\mathrm{ATPase}[64,65]$ and that the $\mathrm{Na}, \mathrm{K}$ ATPase acts as a component of plasma membrane translocation apparatus (PMTA) or regulator of FGF-2 secretion [65] prompted us to search for inhibitors of TCTP/HRF secretion that target putative ATPases on plasma membrane. While screening the possible inhibitors, we found that proton pump inhibitors (PPIs) modulate TCTP/HRF export [20]. Gastric $\mathrm{H}, \mathrm{K}-\mathrm{ATPase}$, mainly found in parietal cells, is an integral membrane protein responsible for gastric acid secretion. It exports $\mathrm{H}^{+}$in exchange for luminal $\mathrm{K}^{+}$, promoting an acidified environment in the stomach, and is thus an factors in acid-related diseases [66]. This led us to postulate that the peptic ulcer drugs, PPIs [67], may have anti-allergic potential, given that they reduces TCTP/HRF secretion [20].

PPIs including omeprazole and pantoprazole block TCTP/HRF secretion from HEK293 and U937 cells, in a concentration-dependent fashion. Also omeprazole and pantoprazole inhibit secretion of both endogenously and exogenously expressed TCTP/HRF. Treatment with ionomycin, a $\mathrm{Ca}^{2+}$ ionophore that induces ER/Golgi-independent secretion, triggers TCTP/HRF secretion in U937 and HEK293 cells, and pre-treatment with omeprazole and pantoprazole abolishes this effect as might be expected [20]. Furthermore, PPIs inhibit the ion transport activity of H,K-ATPase by binding to the catalytic $\alpha$-subunit, and the increased secretion of TCTP/HRF accompanying the overexpression of $\mathrm{H}, \mathrm{K}$ ATPase $\alpha$-subunit, is significantly abolished by pantoprazole treatment. To further confirm these findings, we tested the ability of pantoprazole to inhibit secretion of TCTP/HRF in vivo, using a murine model of ovalbumin (OVA)-induced allergy. Consistent with previous finding that TCTP/HRF is secreted in biological fluids during the late allergic reaction [15-18, 68, 69], TCTP/HRF release was detected in the BAL fluids of OVA-challenged mice [20]. Treatment of pantoprazole remarkably reduced Periodic Acid-Schiff (PAS) positive mucus-producing granular goblet cells, infiltration of inflammatory cells of the lung in a dose-dependent manner, as well as the secretion of TCTP/HRF in BAL fluids, suggesting that pantoprazole exerts anti-allergic effect by blocking TCTP/HRF secretion in vivo [20]. We therefore concluded that omeprazole and pantoprazole exert anti-allergic asthmatic effects by abrogation of TCTP/HRF secretion.

Omeprazole, a specific inhibitor of human gastric $\mathrm{H}, \mathrm{K}$ ATPase [70], has been used in the treatment of peptic ulcer. It is known that omeprazole accumulates in acidic compartments, such as lysosomes, and that the consequent protonation results in an active sulfonamide compound, can act as a potent gastric proton pump inhibitor [71]. In addition, PPIs have also been shown to inhibit a variety of neutrophil functions [72] such as neutrophil-endothelial cell interactions [73, 74], degranulation [72], phagocytosis [75], and production of reactive oxygen intermediates and bactericidal activity [76].

We proposed several possible mechanisms regarding how PPIs act on the $\alpha 1$-subunit of H,K-ATPase and block TCTP/HRF secretion. One possible mechanism is that the activity of putative TCTP/HRF exporting apparatus may be regulated by the electrochemical gradient driven by $\mathrm{H}, \mathrm{K}-$ ATPase. Alternately, based on the observations that PPIs can affect intracellular calcium levels [77, 78], exosomal exit may be another mechanism for TCTP/HRF secretion [19], PPIs perhaps influencing exocytosis and subsequently regulating TCTP/HRF export via modulation of the calcium levels. Finally, though the catalytic $\alpha$-subunit of H,K-ATPase is known as a major target of PPIs, other unknown targets sensitive to PPIs, may also be possibly involved in the secretion mechanism. Our study thus reveals hitherto unknown important function for H,K-ATPase inhibitors. From a clinical perspective, the observation that PPIs avert the secretion of TCTP/HRF which is associated with the aggravation of late phase allergic reaction, obviously underscores the potential of $\mathrm{H}, \mathrm{K}-\mathrm{ATP}$ ase inhibitors as anti-allergic drugs. This possibility needs to be verified.

\section{FACTORS REGULATING THE SECRETION OF TCTP/HRF}

Several lines of evidence suggest that secretion of TCTP/HRF can be influenced by various factors, including specific cytokines such as M-CSF [22], environmental factors including oxidative stress, pollutants such as $\mathrm{H}_{2} \mathrm{O}_{2}$ and reactive oxygen species (ROS)-producing dioxin [21, 23]. 
Teshima et al. observed that macrophage colonystimulating factor (M-CSF) stimulates the synthesis and secretion of a mouse homologue of a human IgE-dependent HRF, by macrophages in vivo and in vivo [22]. They showed that among various macrophage activators tested including M-CSF, GM-CSF, IL-3, tumor necrosis factor (TNF)- $\alpha$, interferon (IFN)- $\gamma$, and lipopolysaccharide (LPS), only M-CSF increased the level of mouse homologue of p26 HRF mRNA and protein, and also stimulated the secretion of an $\mathrm{N}$ glycosylated p26 HRF with m.w. of 30-kDa [22]. They also confirmed the antigen-specific priming of M-CSF in promoting synthesis and secretion of p26 HRF, using cultured macrophages prepared from OVA-immunized mice, and showed the importance of M-CSF in the secretion of p26 $\mathrm{HRF}$ in antigen-sensitized mice [22]. The fact that M-CSF acts as a potent stimulator of induction and secretion of TCTP/HRF in macrophages, underscores its novel role in macrophage functions. These observations indicate that TCTP/HRF released by M-CSF-stimulated macrophage $p e r$ $s e$, plays a pivotal role mediating late phase allergic inflammation.

Since p26 HRF has a potential N-linked glycosylation site (Asp-Xaa-Ser) [79], it was proposed that p26 HRFs of $30-\mathrm{kDa}$ size may be secreted after undergoing posttranslational modifications including $\mathrm{N}$-glycosylation. However, following studies on treatment with $\mathrm{N}$-glycosidase $\mathrm{F}$, of the $30-\mathrm{kDa}$ protein secreted into culture medium, it was concluded that p26 HRF might undergo other complex modifications besides N-glycosylation [22]. Although another cytokine, GM-CSF, has been detected in bronchoalveolar and nasal fluids of late phase reactions [80, 81], where TCTP/HRF was also found [82], TCTP/HRF synthesis and accumulation were found to be insensitive to GM-CSF in macrophages [22].

Besides cytokines, environmental pollutant, dioxin [21], and reactive oxygen species, $\mathrm{H}_{2} \mathrm{O}_{2}$ [23], were shown to trigger TCTP/HRF secretion. Dioxin, 2,3,7,8tetrachlorodibenzo- $p$-dioxin, which is classified as an endocrine disruptor, is one of the most toxic man-made environmental pollutants $[83,84]$. It was found that dioxin induces TCTP/HRF mRNA in mouse embryonic stem (ES) cells via an aryl hydrocarbon receptor (AhR)-dependent pathway and also stimulates the secretion of TCTP/HRF in the culture media of $\mathrm{T}$ and $\mathrm{B}$ cell lines, Jurkat and $\mathrm{BJ} A \mathrm{~B}$ respectively [21]. Dioxin induced synthesis of IgE-dependent HRF is the first example of TCTP/HRF acting as a direct transcriptional target of toxicants [21]. Therefore, endocrine-disrupting agents or environmental pollutants such as dioxin may have the potential to influence allergic disorders in the human via a mechanism involving TCTP/HRF. It is possible that, dioxin affects other physiological conditions by promoting the expression and secretion of TCTP/HRF.

Another study found that low concentrations of $\mathrm{H}_{2} \mathrm{O}_{2}$ trigger TCTP/HRF secretion from BEAS-2B cells [23]. Hydrogen peroxide is a frequently encountered ROS. In studies on the effects of human recombinant HRF (hrHRF) on bronchial epithelium designed to understand the function of TCTP/HRF in airway inflammation, it was found that hrHRF stimulated secretions of IL- 8 and GM-CSF in the primary cultures of human bronchial epithelial cells as well as BEAS-2B cells. Interestingly, $\mathrm{H}_{2} \mathrm{O}_{2}$ but not IL- $1 \beta$ or TNF- $\alpha$, upregulated the synthesis and secretion of TCTP/HRF by bronchial epithelial cells, suggesting that hrHRF stimulates IL- 8 production independently of IL- $1 \beta$ and TNF- $\alpha$. Therefore, oxidative stress may also be a triggering factor for the release of TCTP/HRF from bronchial epithelial cells. Only a secretory form of the $30-\mathrm{kDa} \mathrm{HRF}$, but not an intracellular form of the 26-kDa HRF, was recovered in the culture medium, similar to the previous observations with M-CSF stimulated macrophages [22]. Thus, BEAS-2B cells seem to actively secrete TCTP/HRF under oxidative stress as was the case with mouse ES cells in which TCTP/HRF secretion increased when subjected to ROS-producing dioxin. [21]. All these observations together suggest that environmental pollutants and ROS generated during inflammation, contribute to immune reactions by stimulating TCTP/HRF secretion.

Activated caspase-3 is another TCTP/HRF secretionregulating factor identified, based on the observation that TCTP/HRF export is initiated concurrently with the activation of caspase-3 in serum-starved endothelial cells (EC) [85]. Studies using comparative and functional proteomics, designed to define the mediators released by apoptotic EC, indicated that caspase activation in apoptotic EC promotes the release of antiapoptotic exosome-like nanovesicles and led to the identification of TCTP/HRF as a novel antiapoptotic component of the apoptotic secretome [85]. In addition, it has been shown that increased TCTP/HRF export is a characteristic of apoptotic cell death and that activated caspase- 3 regulates TCTP/HRF export through the nanovesicle pathway [85]. The finding that both chemical inhibition and genetic ablation of caspase- 3 expression prevent TCTP/HRF export, demonstrates the pivotal role of caspase3 in the release of TCTP/HRF-bearing nanovesicles by apoptotic cells [85].

\section{CONCLUDING REMARKS}

To date, functional characterization of TCTP/HRF progressed through studies of intracellular processes as well as extracellular activities [1]. Over the past decade, considerable effort has focused on the role of TCTP/HRF in promoting mediator release from immune cells. This allowed examination of the pathophysiologic events in allergic responses. Although recent studies have also elucidated how and why TCTP/HRF is functionally important to various medically relevant processes, several issues relevant to allergy require further exploration. Further elucidation of key signaling molecules contributing to TCTP/HRF secretion and its regulation may clarify several areas of ambiguity in allergic phenomena and lead to potential use of TCTP/HRF as a therapeutic target. Also, identification of a specific receptor for TCTP/HRF, and characterization of the signaling pathways that activate the cytokine release from cells, are needed to fully understand the role of TCTP/HRF in chronic allergic inflammation. Further studies of the regulation of TCTP/HRF secretion, and development of agents that block the specific receptors involved are also needed. 
Further understanding of the specific role played by TCTP/HRF in allergic phenomena, will lead to development of anti-allergic drugs. From a clinical perspective, since the secretion of TCTP/HRF from cells seems to regulate allergic responses, inhibition of its secretion by drugs such as omeprazole and pantoprazole might be useful approach to antiallergic therapy. In addition, TCTP/HRF has been shown to be pharmacological target for well-known drugs such as sertraline, thioridazine and arteminisin [63]. This inspired us to explore whether these drugs are potentially useful in treating clinical conditions related to the manifold functions of TCTP/HRF. Deciphering the complex functional links of TCTP/HRF with its pleiotropic roles into a unified picture can be accomplished only by discriminating the various molecular forms of TCTP/HRF, and their roles in specific diseases. These efforts are ongoing in many laboratories.

\section{CONFLICT OF INTEERST}

None declared.

\section{ACKNOWLEDGEMENT}

None declared.

\section{REFERENCES}

[1] Bommer UA, Thiele BJ. The translationally controlled tumour protein (TCTP). Int J Biochem Cell Biol 2004; 36: 379-85.

[2] Thaw P, Baxter NJ, Hounslow AM, Price C, Waltho JP, Craven CJ. Structure of TCTP reveals unexpected relationship with guanine nucleotide-free chaperones. Nat Struct Biol 2001; 8: 701-4.

[3] Gachet Y, Tournier S, Lee M, Lazaris-Karatzas A, Poulton T, Bommer UA. The growth-related, translationally controlled protein P23 has properties of a tubulin binding protein and associates transiently with microtubules during the cell cycle. J Cell Sci 1999; 112 (Pt 8): 1257-71.

[4] Kim M, Jung Y, Lee K, Kim C. Identification of the calcium binding sites in translationally controlled tumor protein. Arch Pharm Res 2000; 23: 633-6.

[5] Tuynder M, Susini L, Prieur S, et al. Biological models and genes of tumor reversion: cellular reprogramming through tpt1/TCTP and SIAH-1. Proc Natl Acad Sci U S A 2002; 99: 14976-81.

[6] Li F, Zhang D, Fujise K. Characterization of fortilin, a novel antiapoptotic protein. J Biol Chem 2001; 276: 47542-9.

[7] Kim M, Min HJ, Won HY, et al. Dimerization of translationally controlled tumor protein is essential for its cytokine-like activity. PLoS One 2009; 4: e6464.

[8] MacDonald SM, Rafnar T, Langdon J, Lichtenstein LM. Molecular identification of an IgE-dependent histamine-releasing factor. Science $1995 ; 269$ : 688-90.

[9] Jung J, Kim M, Kim MJ, et al. Translationally controlled tumor protein interacts with the third cytoplasmic domain of $\mathrm{Na}, \mathrm{K}-$ ATPase alpha subunit and inhibits the pump activity in HeLa cells. J Biol Chem 2004; 279: 49868-75.

[10] Kim MJ, Kwon JS, Suh SH, et al. Transgenic overexpression of translationally controlled tumor protein induces systemic hypertension via repression of $\mathrm{Na}+\mathrm{K}+-\mathrm{ATPase}$. J Mol Cell Cardiol 2008; 44: 151-9.

[11] Kim M, Kim HY, Kim S, et al. A protein transduction domain located at the NH2-terminus of human translationally controlled tumor protein for delivery of active molecules to cells. Biomaterials 2011; 32: 222-30.

[12] Fonseca SB, Pereira MP, Kelley SO. Recent advances in the use of cell-penetrating peptides for medical and biological applications. Adv Drug Deliv Rev 2009; 61: 953-64.

[13] Marty C, Meylan C, Schott H, Ballmer-Hofer K, Schwendener RA. Enhanced heparan sulfate proteoglycan-mediated uptake of cellpenetrating peptide-modified liposomes. Cell Mol Life Sci 2004; 61: 1785-94.

[14] Kim M, Chung J, Lee C, Jung J, Kwon Y, Lee K. A peptide binding to dimerized translationally controlled tumor protein modulates allergic reactions. J Mol Med 2011; 89(6): 603-10.
[15] Lichtenstein LM. Histamine-releasing factors and IgE heterogeneity. J Allergy Clin Immunol 1988; 81: 814-20.

[16] Liu MC, Proud D, Lichtenstein LM, et al. Human lung macrophage-derived histamine-releasing activity is due to IgE-dependent factors. J Immunol 1986; 136: 2588-95.

[17] MacDonald SM, Lichtenstein LM, Proud D, et al. Studies of IgEdependent histamine releasing factors: heterogeneity of IgE. J Immunol 1987; 139: 506-12.

[18] Warner JA, Pienkowski MM, Plaut M, Norman PS, Lichtenstein LM. Identification of histamine releasing factor(s) in the late phase of cutaneous IgE-mediated reactions. J Immunol 1986; 136: 25837.

[19] Amzallag N, Passer BJ, Allanic D, et al. TSAP6 facilitates the secretion of translationally controlled tumor protein/histaminereleasing factor via a nonclassical pathway. J Biol Chem 2004; 279: 46104-12.

[20] Choi S, Min HJ, Kim M, Hwang ES, Lee K. Proton pump inhibitors exert anti-allergic effects by reducing TCTP secretion. PLoS One 2009; 4: e5732.

[21] Oikawa K, Ohbayashi T, Mimura J, Fujii-Kuriyama Y, Teshima S, Rokutan K, et al. Dioxin stimulates synthesis and secretion of IgEdependent histamine-releasing factor. Biochem Biophys Res Commun 2002; 290: 984-7.

[22] Teshima S, Rokutan K, Nikawa T, Kishi K. Macrophage colonystimulating factor stimulates synthesis and secretion of a mouse homolog of a human IgE-dependent histamine-releasing factor by macrophages in vitro and in vivo. J Immunol 1998; 161: 6356-66.

[23] Yoneda K, Rokutan K, Nakamura Y, Yanagawa H, KondoTeshima S, Sone S. Stimulation of human bronchial epithelial cells by IgE-dependent histamine-releasing factor. Am J Physiol Lung Cell Mol Physiol 2004; 286: L174-81

[24] Schroeder JT, Lichtenstein LM, MacDonald SM. An immunoglobulin E-dependent recombinant histamine-releasing factor induces interleukin-4 secretion from human basophils. J Exp Med 1996; 183: 1265-70.

[25] Schroeder JT, Lichtenstein LM, MacDonald SM. Recombinant histamine-releasing factor enhances IgE-dependent IL-4 and IL-13 secretion by human basophils. J Immunol 1997; 159: 447-52.

[26] Wantke F, MacGlashan DW, Langdon JM, MacDonald SM. The human recombinant histamine releasing factor: functional evidence that it does not bind to the IgE molecule. J Allergy Clin Immunol 1999; 103: 642-8.

[27] Bheekha-Escura R, MacGlashan DW, Langdon JM, MacDonald SM. Human recombinant histamine-releasing factor activates human eosinophils and the eosinophilic cell line, AML14-3D10. Blood 2000; 96: 2191-8.

[28] Kang HS, Lee MJ, Song H, et al. Molecular identification of IgEdependent histamine-releasing factor as a B cell growth factor. J Immunol 2001; 166: 6545-54.

[29] Orci L, Tagaya M, Amherdt M, et al. Brefeldin A, a drug that blocks secretion, prevents the assembly of non-clathrin-coated buds on Golgi cisternae. Cell 1991; 64: 1183-95.

[30] Tartakoff AM. Perturbation of vesicular traffic with the carboxylic ionophore monensin. Cell 1983; 32: 1026-8.

[31] Nickel W. The mystery of nonclassical protein secretion. A current view on cargo proteins and potential export routes. Eur J Biochem 2003; 270: 2109-19.

[32] Cooper DN, Barondes SH. Evidence for export of a muscle lectin from cytosol to extracellular matrix and for a novel secretory mechanism. J Cell Biol 1990; 110: 1681-91.

[33] Rubartelli A, Cozzolino F, Talio M, Sitia R. A novel secretory pathway for interleukin-1 beta, a protein lacking a signal sequence. EMBO J 1990; 9: 1503-10.

[34] Nickel W. Unconventional secretory routes: direct protein export across the plasma membrane of mammalian cells. Traffic 2005; 6 : 607-14.

[35] Denzer K, Kleijmeer MJ, Heijnen HF, Stoorvogel W, Geuze HJ. Exosome: from internal vesicle of the multivesicular body to intercellular signaling device. J Cell Sci 2000; 113 (Pt 19): 3365-74.

[36] Stoorvogel W, Kleijmeer MJ, Geuze HJ, Raposo G. The biogenesis and functions of exosomes. Traffic 2002; 3: 321-30.

[37] Gould SJ, Booth AM, Hildreth JE. The Trojan exosome hypothesis. Proc Natl Acad Sci U S A 2003; 100: 10592-7.

[38] Raposo G, Nijman HW, Stoorvogel W, et al. B lymphocytes secrete antigen-presenting vesicles. J Exp Med 1996; 183: 1161-72. 
[39] Thery C, Zitvogel L, Amigorena S. Exosomes: composition, biogenesis and function. Nat Rev Immunol 2002; 2: 569-79.

[40] Fevrier B, Raposo G. Exosomes: endosomal-derived vesicles shipping extracellular messages. Curr Opin Cell Biol 2004; 16: 415-21.

[41] Gross B, Gaestel M, Bohm H, Bielka H. cDNA sequence coding for a translationally controlled human tumor protein. Nucleic Acids Res 1989; 17: 8367.

[42] Mignatti P, Morimoto T, Rifkin DB. Basic fibroblast growth factor, a protein devoid of secretory signal sequence, is released by cells via a pathway independent of the endoplasmic reticulum-Golgi complex. J Cell Physiol 1992; 151: 81-93.

[43] Mignatti P, Rifkin DB. Release of basic fibroblast growth factor, an angiogenic factor devoid of secretory signal sequence: a trivial phenomenon or a novel secretion mechanism? J Cell Biochem 1991; 47: 201-7.

[44] Rubartelli A, Sitia R. Interleukin 1 beta and thioredoxin are secreted through a novel pathway of secretion. Biochem Soc Trans 1991; 19: 255-9.

[45] Philip JG, Flower RJ, Buckingham JC. Blockade of the classical pathway of protein secretion does not affect the cellular exportation of lipocortin 1. Regul Pept 1998; 73: 133-9.

[46] Hughes RC. Secretion of the galectin family of mammalian carbohydrate-binding proteins. Biochim Biophys Acta 1999; 1473: 17285 .

[47] Rayne F, Debaisieux S, Bonhoure A, Beaumelle B. HIV-1 Tat is unconventionally secreted through the plasma membrane. Cell Biol Int 2010; 34(4): 409-13.

[48] Ritchie H, Booth NA. Secretion of plasminogen activator inhibitor 2 by human peripheral blood monocytes occurs via an endoplasmic reticulum-golgi-independent pathway. Exp Cell Res 1998; 242: 439-50.

[49] Hogquist KA, Nett MA, Unanue ER, Chaplin DD. Interleukin 1 is processed and released during apoptosis. Proc Natl Acad Sci U S A 1991; 88: 8485-9.

[50] Kuchler K, Thorner J. Secretion of peptides and proteins lacking hydrophobic signal sequences: the role of adenosine triphosphatedriven membrane translocators. Endocr Rev 1992; 13: 499-514.

[51] Muesch A, Hartmann E, Rohde K, Rubartelli A, Sitia R, Rapoport TA. A novel pathway for secretory proteins? Trends Biochem Sci 1990; 15: 86-8.

[52] Lespagnol A, Duflaut D, Beekman C, et al. Exosome secretion, including the DNA damage-induced p53-dependent secretory pathway, is severely compromised in TSAP6/Steap3-null mice. Cell Death Differ 2008; 15: 1723-33.

[53] Ohgami RS, Campagna DR, Greer EL, et al. Identification of a ferrireductase required for efficient transferrin-dependent iron uptake in erythroid cells. Nat Genet 2005; 37: 1264-9.

[54] Steiner MS, Zhang X, Wang Y, Lu Y. Growth inhibition of prostate cancer by an adenovirus expressing a novel tumor suppressor gene, pHyde. Cancer Res 2000; 60: 4419-25.

[55] Amson RB, Nemani M, Roperch JP, et al. Isolation of 10 differentially expressed cDNAs in p53-induced apoptosis: activation of the vertebrate homologue of the drosophila seven in absentia gene. Proc Natl Acad Sci U S A 1996; 93: 3953-7.

[56] Passer BJ, Nancy-Portebois V, Amzallag N, et al. The p53inducible TSAP6 gene product regulates apoptosis and the cell cycle and interacts with Nix and the Myt1 kinase. Proc Natl Acad Sci USA 2003; 100: 2284-9.

[57] Korkmaz KS, Elbi C, Korkmaz CG, Loda M, Hager GL, Saatcioglu F. Molecular cloning and characterization of STAMP1, a highly prostate-specific six transmembrane protein that is overexpressed in prostate cancer. J Biol Chem 2002; 277: 36689-96.

[58] Moldes M, Lasnier F, Gauthereau X, et al. Tumor necrosis factoralpha-induced adipose-related protein (TIARP), a cell-surface protein that is highly induced by tumor necrosis factor-alpha and adipose conversion. J Biol Chem 2001; 276: 33938-46.

[59] Thery C, Boussac M, Veron P, et al. Proteomic analysis of dendritic cell-derived exosomes: a secreted subcellular compartment distinct from apoptotic vesicles. J Immunol 2001; 166: 7309-18.

[60] Luketic L, Delanghe J, Sobol PT, et al. Antigen presentation by exosomes released from peptide-pulsed dendritic cells is not suppressed by the presence of active CTL. J Immunol 2007; 179: 5024-32.

[61] Yu X, Harris SL, Levine AJ. The regulation of exosome secretion: a novel function of the p53 protein. Cancer Res 2006; 66: 4795801.
[62] Levine AJ. p53, the cellular gatekeeper for growth and division. Cell 1997; 88: 323-31.

[63] Telerman A, Amson R. The molecular programme of tumour reversion: the steps beyond malignant transformation. Nat Rev Cancer 2009; 9: 206-16.

[64] Dahl JP, Binda A, Canfield VA, Levenson R. Participation of $\mathrm{Na}, \mathrm{K}-\mathrm{ATPase}$ in FGF-2 secretion: rescue of ouabain-inhibitable FGF-2 secretion by ouabain-resistant Na,K-ATPase alpha subunits. Biochemistry 2000; 39: 14877-83.

[65] Florkiewicz RZ, Anchin J, Baird A. The inhibition of fibroblast growth factor-2 export by cardenolides implies a novel function for the catalytic subunit of Na+,K+-ATPase. J Biol Chem 1998; 273: 544-51.

[66] Shin JM, Munson K, Vagin O, Sachs G. The gastric HK-ATPase: structure, function, and inhibition. Pflugers Arch 2009; 457: 60922.

[67] Bell NJ, Hunt RH. Role of gastric acid suppression in the treatment of gastro-oesophageal reflux disease. Gut 1992; 33: 118-24.

[68] Budde IK, Lopuhaa CE, de Heer PG, et al. Lack of correlation between bronchial late allergic reaction to Dermatophagoides pteronyssinus and in vitro immunoglobulin $\mathrm{E}$ reactivity to histamine-releasing factor derived from mononuclear cells. Ann Allergy Asthma Immunol 2002; 89: 606-12.

[69] Pasmans SG, Witteman AM, Aalbers M, et al. Variability of IgEdependent histamine-releasing activity in supernatants of human mononuclear cells. Int Arch Allergy Immunol 1994; 103: 44-52.

[70] Im WB, Sih JC, Blakeman DP, McGrath JP. Omeprazole, a specific inhibitor of gastric (H+-K+)-ATPase, is a H+-activated oxidizing agent of sulfhydryl groups. J Biol Chem 1985; 260: 4591-7.

[71] Lorentzon P, Jackson R, Wallmark B, Sachs G. Inhibition of (H++ $\mathrm{K}+$ )-ATPase by omeprazole in isolated gastric vesicles requires proton transport. Biochim Biophys Acta 1987; 897: 41-51.

[72] Wandall JH. Effects of omeprazole on neutrophil chemotaxis, super oxide production, degranulation, and translocation of cytochrome b-245. Gut 1992; 33: 617-21.

[73] Suzuki M, Mori M, Fukumura D, Suzuki H, Miura S, Ishii H. Omeprazole attenuates neutrophil-endothelial cell adhesive interaction induced by extracts of Helicobacter pylori. J Gastroenterol Hepatol 1999; 14: 27-31.

[74] Yoshida N, Yoshikawa T, Tanaka Y, et al. A new mechanism for anti-inflammatory actions of proton pump inhibitors--inhibitory effects on neutrophil-endothelial cell interactions. Aliment Pharmacol Ther 2000; 14 (Suppl 1): 74-81.

[75] Agastya G, West BC Callahan JM. Omeprazole inhibits phagocytosis and acidification of phagolysosomes of normal human neutrophils in vitro. Immunopharmacol Immunotoxicol 2000; 22: 357-72.

[76] Zedtwitz-Liebenstein K, Wenisch C, Patruta S, Parschalk B, Daxbock F, Graninger W. Omeprazole treatment diminishes intra- and extracellular neutrophil reactive oxygen production and bactericidal activity. Crit Care Med 2002; 30: 1118-22.

[77] Grant RL, Acosta D. Interactions of intracellular $\mathrm{pH}$ and intracellular calcium in primary cultures of rabbit corneal epithelial cells. In vitro Cell Dev Biol Anim 1996; 32(1): 38-45.

[78] Holm A, Sundqvist T, Oberg A, Magnusson KE. Mechanical manipulation of polymorphonuclear leukocyte plasma membranes with optical tweezers causes influx of extracellular calcium through membrane channels. Med Biol Eng Comput 1999; 37: 410-2.

[79] Chitpatima ST, Makrides S, Bandyopadhyay R, Brawerman G. Nucleotide sequence of a major messenger RNA for a 21 kilodalton polypeptide that is under translational control in mouse tumor cells. Nucleic Acids Res 1988; 16: 2350.

[80] Durham SR, Ying S, Varney VA, et al. Cytokine messenger RNA expression for IL-3, IL-4, IL-5, and granulocyte/macrophagecolony-stimulating factor in the nasal mucosa after local allergen provocation: relationship to tissue eosinophilia. J Immunol 1992; 148: 2390-4.

[81] Robinson DS, Hamid Q, Ying S, et al. Predominant TH2-like bronchoalveolar T-lymphocyte population in atopic asthma. N Engl J Med 1992; 326: 298-304.

[82] MacDonald SM. Histamine-releasing factors. Curr Opin Immunol 1996; 8: 778-83.

[83] Chapman DE, Schiller CM. Dose-related effects of 2,3,7,8tetrachlorodibenzo-p-dioxin (TCDD) in C57BL/6J and DBA/2J mice. Toxicol Appl Pharmacol 1985; 78: 147-57.

[84] McGregor DB, Partensky C, Wilbourn J, Rice JM. An IARC evaluation of polychlorinated dibenzo-p-dioxins and polychlori- 
nated dibenzofurans as risk factors in human carcinogenesis. Environ Health Perspect 1998; 106 (Suppl 2): 755-60.
[85] Sirois I, Raymond MA, Brassard N, et al. Caspase-3-dependent export of TCTP: a novel pathway for antiapoptotic intercellular communication. Cell Death Differ 2011; 18: 549-62.

Received: January 25, 2012

(C) Maeng et al.; Licensee Bentham Open.

This is an open access article licensed under the terms of the Creative Commons Attribution Non-Commercial License (http://creativecommons.org/licenses/by-nc/3.0/) which permits unrestricted, non-commercial use, distribution and reproduction in any medium, provided the work is properly cited. 